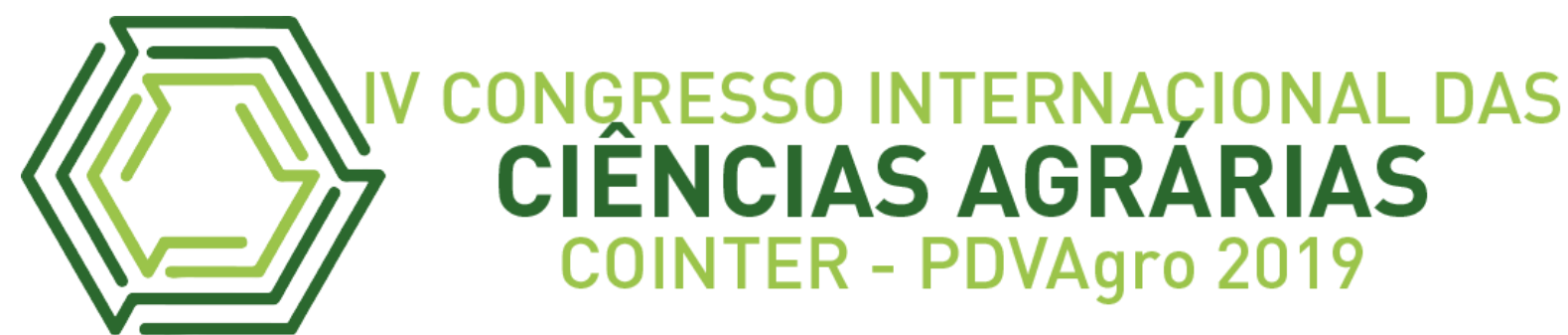

\title{
COMPREENDENDO O PADRÃO DE PRECIPITAÇÃO USANDO ANÁLISES DE DADOS FUNCIONAIS
}

\section{COMPREENDIENDO EL PATRÓN DE PRECIPITACIÓN USANDO ANÁLISIS DE DATOS FUNCIONALES}

\section{UDERSTANDING PRECIPTATION PATTER USING FUNCTIOAL DATA ANALYSIS}

\author{
Apresentação: Comunicação Oral
}

Mickaelle Maria de Almeida Pereira ${ }^{1}$; Leika Irabele Tenório de Santana ${ }^{2}$; Jucarlos Rufino de Freitas $^{3}$; Joelma Mayara da Silva ${ }^{4}$ Paulo José Duarte Neto ${ }^{5}$

\section{DOI: https://doi.org/10.31692/2526-7701.IVCOINTERPDVAgro.2019.0038}

\begin{abstract}
Resumo
A precipitação é um dos fatores que mais se destacam para determinar o clima de uma região, essa variável proveniente de um sistema climático complexo, possui alta variabilidade local e é objeto de diversos estudos. Diante disto, este trabalho tem como objetivo estudar as principais fontes de variações e determinar os comportamentos padronizados entre diferentes curvas funcionais das séries de acúmulo de precipitação mensal dos municípios de Recife e Vitória de Santo Antão no período de janeiro de 2000 a dezembro de 2018, disponibilizado pela Agência Pernambucana de Águas e Climas (APAC), utilizando análise de dados funcionais (FDA). A FDA possibilita reestruturar os dados de forma contínua sem perder a variabilidade das informações. A base de Fourier foi utilizada para suavizar os dados, pois eles têm um comportamento periódico. A análise de componente principal funcional (FPCA) mostrou grande variação entre os meses de maio, junho e julho, que estão relacionado aos meses de inverno e houve uma pequena variação para os meses de janeiro e fevereiro decorrentes das tempestades de verão, onde estão associadas ao comportamento climático dos municípios. A análise de cluster separou os funcionais em grupos de acordo com suas similaridades, que foi verificado a confirmação dos grupos por meio do Biplot do FPCA. A análise de variância funcional (FANOVA) comparou as médias do acúmulo de precipitação mensal, mesmo os municípios estarem relativamente próximo a FANOVA constatou diferença significativa $(p<0,01)$ entre suas médias. Portanto, a análise de dados funcionais demostrou uma forte flexibilidade em estudar os comportamentos das observações mostrando que com um menor quantitativo de informações, pode-se extrair resultados relevantes para o fenômeno.
\end{abstract}

\footnotetext{
1 Programa de Pós-graduação em Biometria e Estatística Aplicada, UFRPE, e-mail: mickaellealmeida1@gmail.com

${ }^{2}$ Programa de Pós-graduação em Biometria e Estatística Aplicada, UFRPE, e-mail: leikatenorio10@gmail.com

3 Programa de Pós-graduação em Biometria e Estatística Aplicada, UFRPE, e-mail: jucarlos123@hotmail.com

${ }^{4}$ Programa de Pós-graduação em Biometria e Estatística Aplicada, UFRPE, e-mail: jms.estat@gmail.com

${ }^{5}$ Doutor em Biometria e Estatística Aplicada, UFRPE, e-mail: pjduarteneto@gmail.com
} 
Palavras-Chave: Chuva, Dinâmica, FDA, Variação.

\title{
Resumen
}

La precipitación es uno de los factores que más se destacan para determinar el clima de una región, ésta variable provenientes de un sistema climático complejo, con alta variabilidad local, es objeto de diferentes estudios. Debido a esto, este trabajo tiene como objetivo, estudiar las principales fuentes de variación y determinar los comportamientos estandarizados entre diferentes curvas funcionales de las series de acumulación de precipitación mensual de los municipios de Recife y Vitória de Santo Antão, desde enero de 2000 hasta diciembre de 2018, disponibles en la Agencia de Agua y Clima de Pernambuco (APAC), utilizando análisis de datos funcionales (FDA). El FDA hace posible reestructurar continuamente los datos sin perder la variabilidad de la información. La base de Fourier fue utilizada para suavizar los datos, ya que tienen un comportamiento periódico. El análisis del componente funcional principal (FPCA) mostró una gran variación entre los meses de mayo, junio y julio, que están relacionados con los meses de invierno, además de presentar una ligera variación en los meses de enero y febrero debido a las tormentas de verano, donde están asociados al comportamiento climático de los municipios. El análisis de aglomerados separó los grupos funcionales en grupos de acuerdo con sus similitudes, lo que confirmó que los grupos usaban FPCA Biplot. El análisis de varianza funcional (FANOVA) comparó las medias de acumulación de precipitación mensual, a pesar que los municipios estaban relativamente cerca de FANOVA encontraron diferencias significativas $(\mathrm{p}<0.01)$ entre sus promedios. Por lo tanto, el análisis de los datos funcionales mostró una gran flexibilidad en el estudio de los comportamientos de las observaciones que muestran que, con una menor cantidad de información, se pueden extraer resultados relevantes para el fenómeno.

Palabras Clave: Lluvia, Dinámica, FDA, Variación.

\begin{abstract}
Precipitation is one of the most prominent factors in determining the climate of a region. This variable generated by a complex climate system, with high local variability, is the subject of several studies. Given this, this paper aims to study the main sources of variation and to determine the standardized behaviors between different functional curves of the monthly precipitation accumulation series of the municipalities of Recife and Vitória de Santo Antão from January 2000 to December 2018, available from the Pernambuco Water and Climate Agency (APAC), using functional data analysis (FDA). The FDA makes it possible to continuously restructure data without losing information variability. The Fourier base was used to smooth the data because it has a periodic behavior. The principal functional component analysis (FPCA) showed great variation between the months of May, June and July, which are related to the winter months and there was a slight variation for the months of January and February due to the summer storms, where they are associated. to the climatic behavior of the municipalities. Cluster analysis separated the functional groups into groups according to their similarities, which confirmed the groups using the FPCA Biplot. The analysis of functional variance (FANOVA) compared the means of monthly precipitation accumulation, even though the municipalities were relatively close to FANOVA found significant difference $(\mathrm{p}<0.01)$ between their averages. Therefore, the analysis of functional data showed a strong flexibility in studying the behaviors of observations showing that with a smaller amount of information, relevant results for the phenomenon can be extracted.
\end{abstract}


Keywords: Rainfall, Dynamic, FDA, Variation.

\section{Introdução}

Vários fatores podem determinar o clima de uma região, entre eles destacam-se: a circulação geral da atmosfera, a topografia local, a cobertura vegetal, o ciclo hidrológico (ou ciclo da água) e as influências das correntes marítimas, que agem tanto na escala global quanto regional (MOLION, 1987). Consequentemente, a variabilidade climática pode acarretar graves alterações na vida econômica e social de uma região, como por exemplo na agricultura, no fornecimento de energia, no ciclo turístico e indiretamente no setor produtivo (DE LUCENA; STOSIC; FILHO, 2015). Uma das variáveis mais relevantes para adquirir um melhor entendimento sobre as condições climáticas de uma determinada região é a precipitação (ALMAZROUI et al. 2012).

A precipitação é um recurso natural que contribui para o abastecimento das bacias hidrográficas, auxiliando no ciclo hidrológico da água, contudo, índices elevados de chuva podem trazer consequências para a população, tais como alagamentos, enchentes e inundações que afetam a infraestrutura de uma determinada região.

No Brasil, análises de mudanças climáticas locais vem atraindo o interesse de diversos pesquisadores, baseando-se na estimativa dos índices de detecção de mudanças climáticas da Organização Meteorológica Mundial (OMM) (DOS SANTOS et al., 2006; SILVA; DERECZYNSKI, 2010; ALMEIDA; REBELLO; AMBRIZZI, 2010). A partir Santos (2006) e Silva et al. (2006), iniciou-se com mais intensidade análises em escalas locais das tendências de índices de extremos climáticos no Nordeste brasileiro. O estado de Pernambuco, situado no Nordeste brasileiro apresenta uma ampla variabilidade pluviométrica, porém, poucos estudos referentes a índices de extremos climáticos foram desenvolvidos (SOUZA; AZEVEDO, 2012; SILVA; MONTENEGRO; SOUZA, 2017).

Neste sentido, busca-se estudar de maneira mais detalhada a precipitação em duas cidades, Recife capital do estado de Pernambuco situada na mesorregião metropolitana do estado e Vitória de Santo Antão, situada na Zona da Mata do estado, através da análise de dados funcional (Functional Data Analysis - FDA), com intuito de estudar as principais fontes de variações e determina os comportamentos padronizados entre diferentes curvas funcionais, pois, tratam-se de cidades sensíveis a evento extremos de seca e de alta pluviosidade em curto espaço de tempo, Recife por conta do crescimento populacional em áreas de risco e Vitória de Santo Antão por conta do rio Tapacurá que banha a cidade. 


\section{Fundamentação Teórica}

Séries de precipitação foram o objeto de diversos estudos que se diferenciam em objetivo e técnicas. Andreoli e Kayano (2007) e Lucena, Cervain e Gomes Filho (2011) estudaram o efeito dos eventos El Niño e La Niña na variabilidade da precipitação no nordeste brasileiro e a influência da temperatura da superfície do mar nas alterações climáticas.

Técnicas da estatística foram utilizadas, buscando explicar a variabilidade temporal (SILVA et al., 2010; SALGADO et al., 2007) e tendência (SILVA et al., 2010; SOUZA NETO, 2017; SILVA, MONTENEGRO, SOUZA, 2017).

Técnicas da Física estatística foram utilizadas devido à complexidade do sistema climático. Xavier Jr. et al. (2019) utilizaram a técnica Sample Entropy para avaliar a complexidade das séries de precipitação do estado da Paraíba e verificaram que há diferença nas precipitações médias mensais das mesorregiões do estado, além disso através de suas análises foi possível verificar que existem variações pluviométricas locais. De Lucena, Stosic e Cunha Filho (2015) avaliaram a variabilidade temporal da precipitação diária através da técnica de lacunaridade.

Entre as dificuldades muitas vezes encontradas pelos pesquisadores estão relacionadas a complexidade das séries e a alta incidências de zero, pois, no caso de técnicas da estatística é necessário utilizar modelos adequados a alta incidência de zeros e para os métodos da física estatística é necessário cerca de 30 anos de observações, diante disto, propomos utilizar a análise de dados funcionais para qual não é necessário um grande número de observações e o acumulado mensal de precipitação, não tendo grande incidência de zeros.

\section{Análise de dados funcionais}

A análise de dados funcionais, conhecida como FDA foi introduzida na década de 90 através de Ramsay e Dalzell (1991) a partir de seu trabalho intitulado Some tools for functional data analysis, onde eles representam FDA como uma função $x_{i}(t), i=1, \ldots, t$, $t \in T$, onde $T$ é um intervalo real . A FDA foi empregada em diversas áreas de pesquisas, nas ciências médicas (RAMSAY; BOCK; GASSER, 1995; DONA et al. 2009), nas emissões de gases do efeito estufa (GAO, 2007; GAO, NIEMEIER, 2008) e nas ciências econômicas (WANG et al., 2008), entre outras. Ao invés de pontos no tempo único, cada função, produzida a partir de uma série de observações sucessivas, é analisada como uma só 
observação, possibilitando a retirada de informações de um procedimento temporal como um todo

\section{Suavização}

O acúmulo mensal de precipitação por natureza é um processo contínuo ao longo do tempo, mas gravados em pontos discretos de $t \in T=[1,12]$. Primeiro vamos reestruturar nossos dados discretos, acúmulo de precipitação mensal para a cidade de Recife e Vitória de Santo Antão - PE, como um dado funcional, para esse processo precisamos utilizar alguma técnica de suavização, como expansão de base (RAMSAY; SILVERMAN, 1997), definida como:

$$
p(t)=\sum_{k=1}^{K} a_{k} \emptyset_{k}(t)
$$

onde $\emptyset_{k}(t)$ é o conjunto de funções bases combinadas linearmente, e os $a_{k}^{\prime} s$ são os coeficientes de expansão. Para encontrar as funções bases podemos usar o alisamento Bspline, de acordo com Ullah e Finch (2013) a suavização B-spline é a mais popular técnica utilizada, e em segundo lugar a base de Fourier. A base de Fourier é empregada em processos periódicos, a mesma consiste em funções $1, \sin \omega t, \cos \omega t, \sin 2 \omega t, \cos 2 \omega t, \ldots$ onde $\omega=\frac{2 \pi}{T}$ e $T=12$ que é o período mensal (RAMSAY; HOOKER; GRAVES, 2009).

\section{Análise de componentes principais funcionais}

A análise de componentes principais (FPCA) é um estudo multivariado mais conhecida para retirar informações de observações funcionais (CROUX; GAZEN, 2005; ULLAH; FINCH, 2013), pois, queremos examinar quais são as principais formas, modos de variação que estão nos dados (RAMSAY; HOOKER; GRAVES, 2009). Esse estudo diminui as dimensões de um conjunto de dados onde existe um amplo número de variáveis interrelacionadas, ao mesmo tempo, ele ainda retém a maior variação total existente. Essa diminuição é realizada através da transformação dos dados para outro conjunto de variáveis, ou componentes principais, que não são mais inter-relacionados (ULLAH; FINCH, 2013). Os componentes principais, em FPCA, é definido por uma função $\xi$ conhecida como função peso, ou como função harmônica, delimitada sobre o mesmo intervalo de tempo, $T$. Da mesma forma que o PCA, o FPCA pode ser interpretado individualmente (GAO; NIEMEIER, 2008). Desta forma temos, 


$$
y_{i}=\int \xi(t) p_{i}(t) d t
$$

onde a função peso está associado aos dados funcionais, mas para isso ocorrer precisa-se que haja uma restrição em relação ao tamanho de $\xi$, onde $\int \xi^{2}(t) d t$ (RAMSAY; HOOKER; GRAVES, 2009). Cada componente principal representa uma quantidade de porcentagem da variabilidade os objetos de dados funcionais (RAMSAY, 2004).

\section{Análise de variância funcional}

A análise de variância funcional (FANOVA) oferece opções, aos métodos da análise de variância clássica. Considere os conjuntos de $z$ de funções aleatórias independentes $X_{i j}(t)$, $i=1, \ldots$, z e $j=1, \ldots, n_{i}$ definidas em um período limitado e fechado $I=[a, b]$. E $n=$ $n_{1}+\cdots+n_{z}$. Esses conjuntos podem-se diferenciar em relação as funções médias, ou seja, assumimos que $X_{i j}(t)$ são processos estocásticos com função média $\mu_{i}(t), t \in I$, e função de covariância $\gamma(s, t), s, t \in I$. Também assumimos $X_{i j} \in L_{2}(I), i=1, \ldots, z, j=1, \ldots, n_{i}$, onde $L_{2}(I)$ está definido no espaço de Hilbert que compõe-se em funções integráveis e quadradas em $I$, equipado com o produto interno da forma $\langle f, g\rangle=\int_{I} f(t) g(t) d t$. O interesse é testar a seguinte hipótese nula

$$
H_{0}: \mu_{1}(t)=\cdots=\mu_{z}(t), t \in I
$$

Há hipótese alternativa $\left(H_{a}\right)$ é a negação da $H_{0}$ (GÓRECKI; SMAGA, 2019).

\section{Análise de cluster funcional}

Uma interpretação à análise de cluster é buscar por grupos naturais de observações considerando distância entre as observações (CLARKSON et al., 2005).

Com as matrizes de distância calculadas a partir de dados funcionais do acúmulo mensal de precipitação, qualquer técnica de agrupamento (por exemplo, agrupamento hierárquico) pode ser utilizado para criar as aglomerações (GAO; NIEMEIER, 2008). O agrupamento hierárquico é uma interpretação algorítmica, empregando modos de aglomeração ou divisão, que requer uma medida diferente entre grupos de observações, que diz quais clusters podem ser combinados ou quando um cluster pode ser dividido. Os centros de cluster visam separar as observações em $k$ aglomerados, de forma que a soma das distâncias quadradas dentro do cluster, centrada nos meios, seja minimizada (WANG; CHIOU; MÜLLER, 2016). 


\section{Metodologia}

\section{Caracterização da área}

Os Municípios de Recife e Vitória de Santo Antão situam-se no estado de Pernambuco (Figura 1), localizado na NEB, de coordenadas geográficas $8^{\circ} 03^{\prime} 14^{\prime \prime}$ latitude Sul e $34^{\circ} 52^{\prime}$ 53" longitude Oeste (Recife), $8^{\circ}$ 07' 05" latitude Sul e 35 17' 29" longitude Oeste (Vitória de Santo Antão). As regiões ocupam uma área de $218,843 \mathrm{~km}^{2}$ e $335,941 \mathrm{~km}^{2}$ com população estimada de 1.637 .834 e 137.915 habitantes (disponível em: https://www.ibge.gov.br. Acessado em 27/08/2019).

Figura 1: Espacialização geográfica dos municípios de Recife e Vitória de Santo Antão, PE, Brasil.

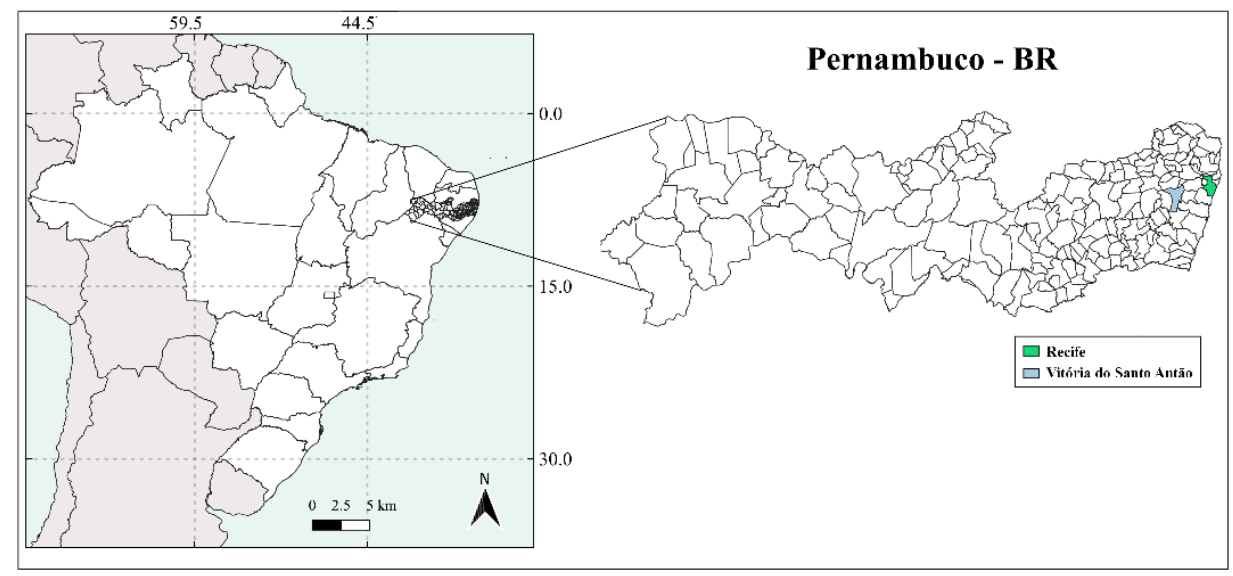

Fonte: Própria (2019).

\section{Dados}

O conjunto de informações utilizadas para o desenvolvimento do trabalho refere-se aos registros pluviométricos do posto de monitoramento Posto 26 - IMA, localizado no município de Vitória de Santo Antão - PE e posto de monitoramento Posto 30 - Várzea, localizado no município de Recife, que foram disponibilizados pela Agência Pernambucana de Águas e Climas (APAC), no período de janeiro de 2000 a dezembro de 2018. Na base inicial de dados constavam dez (Recife) e nove (Vitória de Santo Antão) postos de monitoramento, onde foi selecionado um posto de cada município, pois os demais não apresentavam os dados completos, inviabilizando a análise.

$\mathrm{Na}$ etapa inicial, os dados foram suavizados através do alisamento de Fourier, em seguida aplicou-se análise componente principal funcional (FPCA) para analisar as variações 
funcionais. Posteriormente, a análise de cluster para agrupar as funções de acordo com a similaridade entre elas, e por fim, foi introduzido a análise de variância funcional (FANOVA) para verificar se existe diferença significativa entre os municípios. Todas as análises foram realizadas no software R (R CORE TEAM, 2019).

\section{Resultados e Discussão}

A Figura 2, representa a reestruturação dos dados brutos do acúmulo mensal de precipitação, referentes ao posto 30 do município de Recife e ao posto 26 do município de Vitória de Santo Antão - PE, durante o período de janeiro de 2000 a dezembro de 2018. Foram utilizadas onze funções bases de Fourier produzindo um nível considerável de precisão e suavidade, após essa transformação obteve-se uma função completa e contínua do acúmulo de precipitação mensal para os dois munícipios, assim, permitindo as análises estatísticas, conservando a variabilidade nos dados originais.

Com base na Figura 2, verifica-se que maio, junho e julho são os meses mais chuvosos de Recife (Figura (a)) e Vitória de Santo Antão (Figura (b)), com uma média máxima de aproximadamente $180 \mathrm{~mm}$ e $380 \mathrm{~mm}$, com um mínimo mensal de zero mm e um máximo mensal de aproximadamente $600 \mathrm{~mm}$. A razão para os altos indices de chuva no meio pode ser devido ao fenômeno La Niña, um intensificador de chuvas na Amazônia, no Nordeste e em partes do Sudeste combinada com o inverno na região, contudo, em 2012 houve um baixo índice pluviométrico de aproximadamente $100 \mathrm{~mm}$ e $210 \mathrm{~mm}$ menor do que média em relação ao inverno, este resultado provém do fenômeno El Niño, onde os municípios sofreram forte influência dos padrões anômalos da temperatura da superfície do mar (ATSM) (ANDREOLI et al., 2004, NÓBREGA; SANTIAGO, 2014). O excesso ou falta presumivelmente afetam de forma direta ou indireta toda a cadeia produtiva agrícola ou pecuária dos municípios.

Ao analisar as funções de dados funcionais (Figura 2) foi observado que o município do Recife apresentou maiores variações de precipitação em relação a Vitória do Santo Antão, estas variações estão relacionadas com a localização geográfica do município localizado no litoral onde sofrem variações climáticas por conta de eventos extremos. 
Figura 2: Dados suavizados do acúmulo mensal da precipitação de Recife (a) e Vitória de Santo Antão (b) no período de 2000 a 2018, Pernambuco, Brasil.
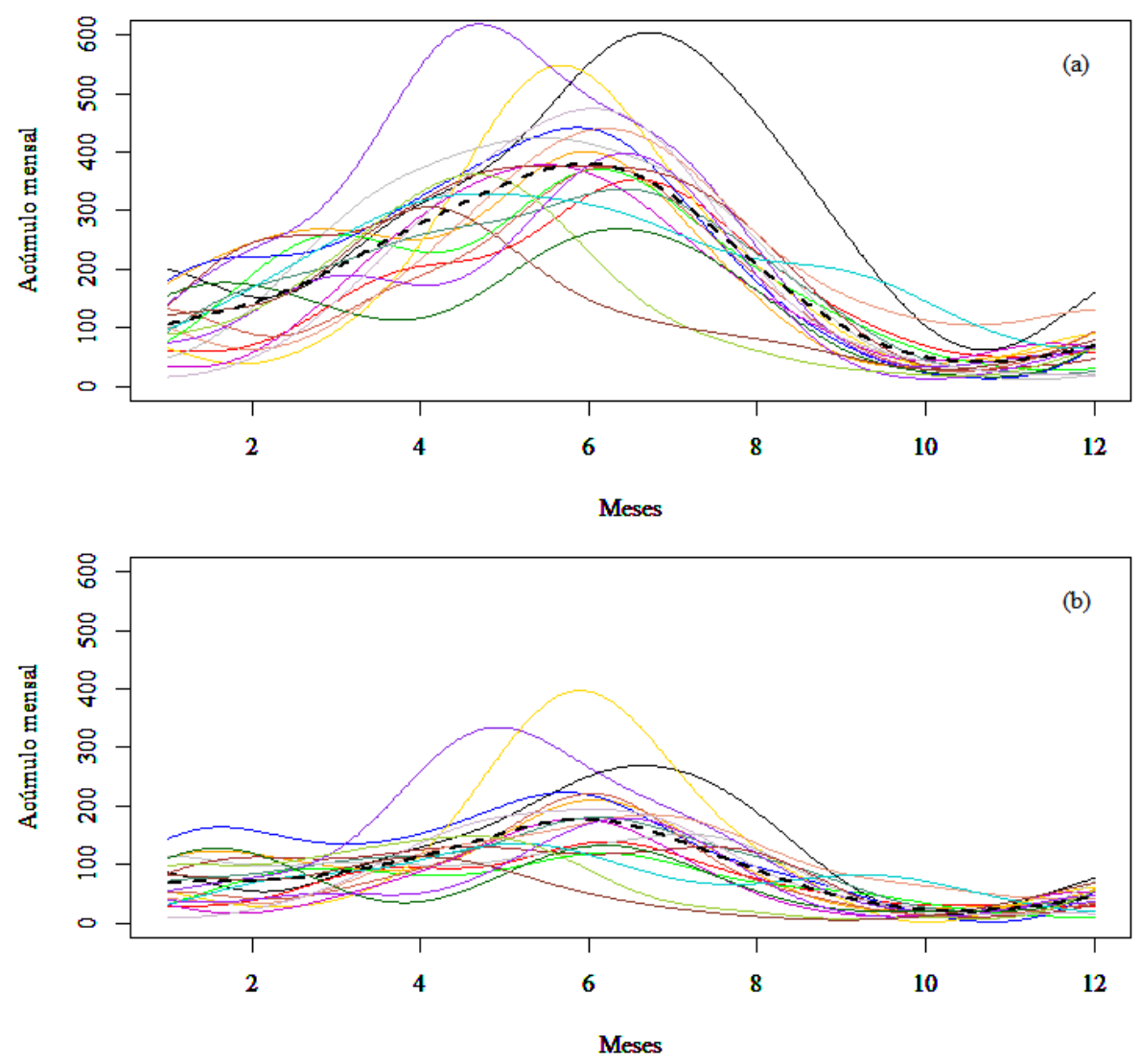

Fonte: Própria (2019).

As Figuras 3 e 4 representam a análise dos componentes principais funcionais (FPCA) , observa-se a curva média global da precipitação, bem como duas curvas adicionais para cada PC. Essas duas curvas adicionais mostram o que acontece com a curva média quando um desvio padrão harmonico é adicionado (+) ou subtraído (-). Dessa forma, constata-se que o primeiro harmônico (Figura 3) capturou $54,5 \%$ do total variação sobre a média. O segundo harmônico, explica um adicional de 25,9\% de variação, indicou uma mudança vertical na média, totalizando $80,4 \%$ da variação. O terceiro harmônico capturou $11,4 \%$ os desvios verticais, e os harmônicos restantes capturaram ruído e variação muito menores. Analogamente, a FPCA (Figura 4) representa $65,9 \%$ e o segundo $16,7 \%$ totalizando $82,6 \%$ da variação. Um estudo extensivo realizado por Rencher (2002), afirma que se o primeiro e 
segundo componentes principais explicam no mínimo $70 \%$ da variação total dos dados pode ser feita uma análise sobre o fenômeno, por isso, o estudo delimita-se ao primeiro e segundo

Figura 3: $1^{\circ}$ e $2^{\circ}$ FPCA correspondente ao município de Recife, no período de 2000 a 2018, Pernambuco, Brasil.

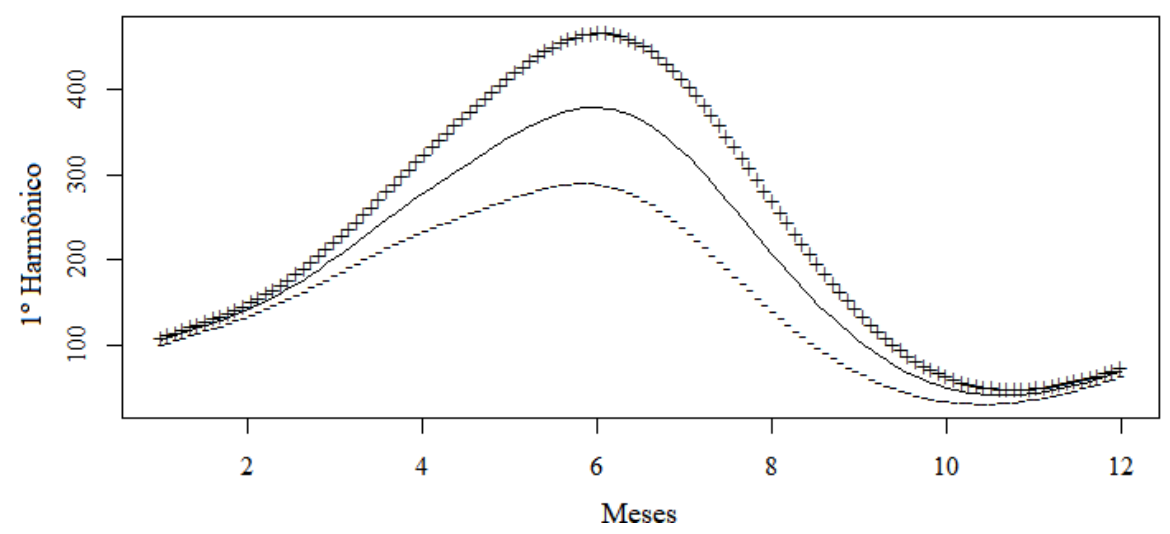

componentes principais.

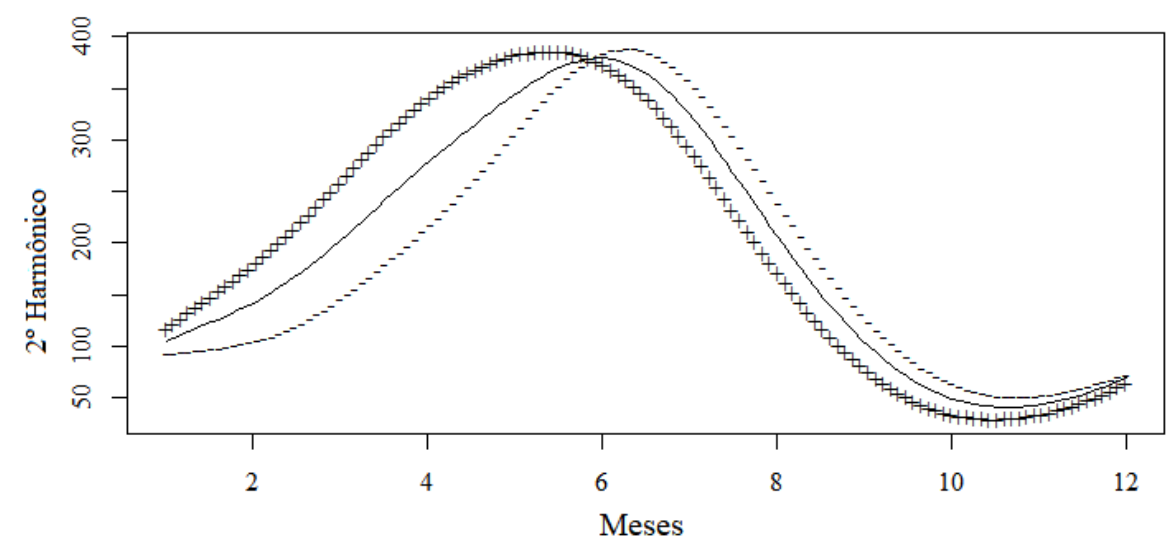

Fonte: Própria (2019). 
Figura 4: $1^{\circ}$ e $2^{\circ}$ FPCA correspondente ao município de Vitória de Santo Antão, no período de 2000 a 2018 , Pernambuco, Brasil.
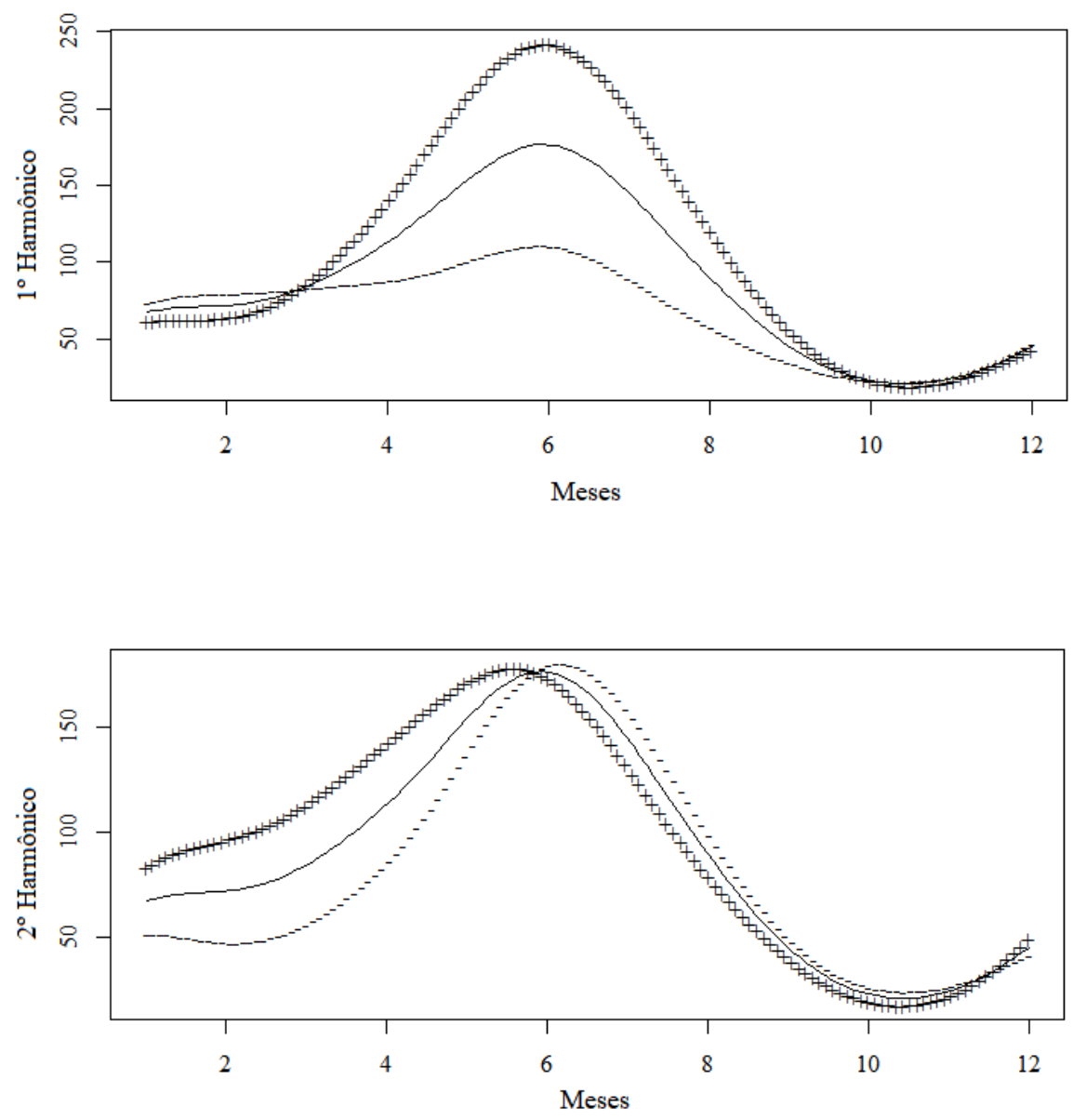

Fonte: Própria (2019).

Analisando os agrupamentos para o município de Vitória de Santo Antão (Figura 5), observa-se que a análise de cluster separou os anos em cinco grupos com base nas similaridades dos anos. Para o primeiro grupo (2005 e 2011) ocorreram maiores precipitações significativas todas acima da média, sendo considerado um outlier na amostra, esse resultado está associado as enchentes ocorridas neste período (DE SANTANA et al., 2019; MIRANDA, 2015). De maneira análoga, para o segundo grupo (2016 e 2018) o processo foi inverso, ocorrendo períodos secos prejudicando o setor agrícola da região. Já o terceiro grupo (2000 e 2004), o comportamento das curvas funcionais evidenciaram um crescimento um pouco acima da média entre os meses março a outubro, porém, no grupo quatro (2001, 2003, 2012 e 2014), o processo foi oposto ao descrito anteriormente. No último grupo, o acúmulo mensal conseguiu captar o comportamento da curva média apresentando poucas variações, ou seja, 
houve uma sobreposição quase perfeita em cima da média em relação aos outros grupos propriamente dito.

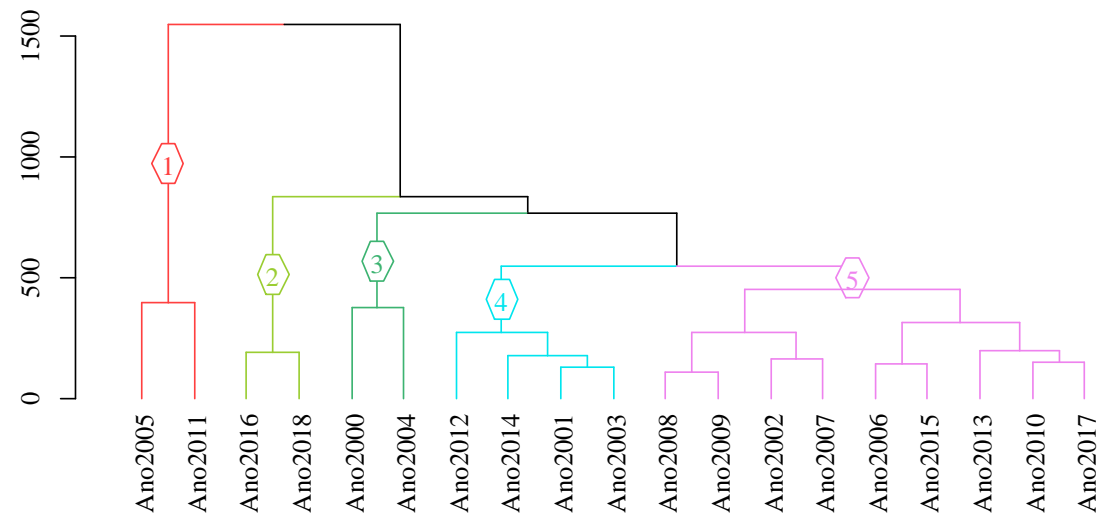

Fonte: Própria (2019).

A Figura 6, retrata o Biplot do $1^{\circ}$ FPCA vs $2^{\circ}$ FPCA, em que apresenta os comportamentos dos grupos através da sua variabilidade, assim, observa-se que o resultado corresponde ao apresentado na análise de cluster.

Figura 6: Biplot do FPCA correspondente ao município de Vitória de Santo Antão, no período de 2000 a 2018, Pernambuco, Brasil.

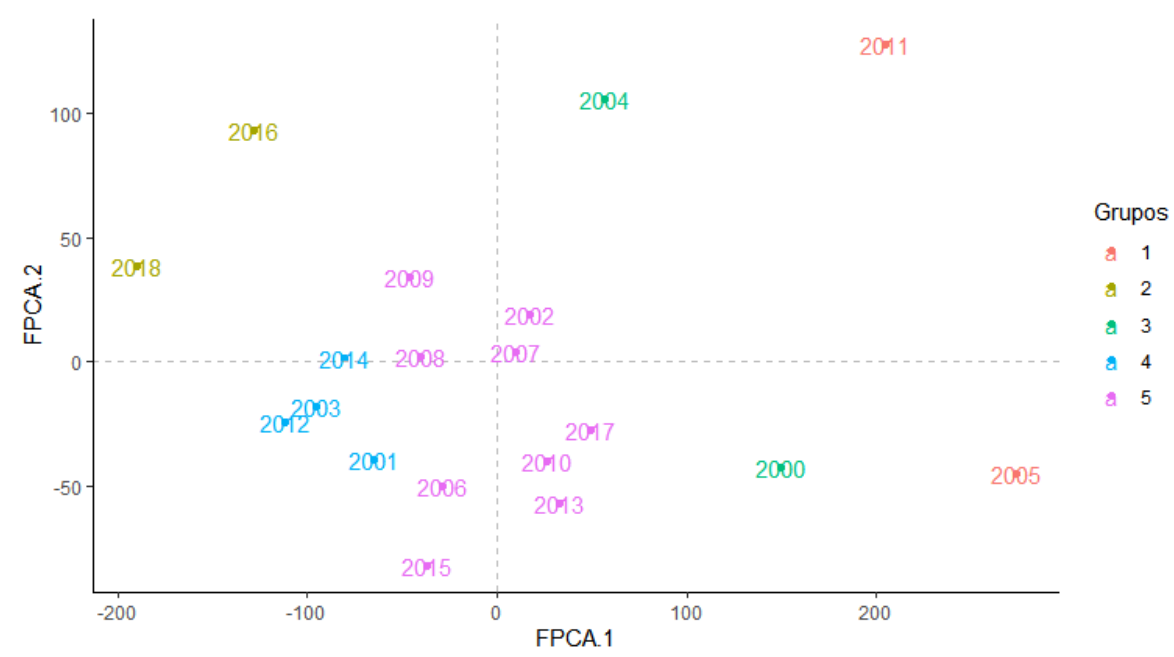

Fonte: Própria (2019).

A Figura 7 e 8 apresentam o cluster e o Biplot do FPCA correspondente ao munícipio de Recife, nesse observa-se cinco diferentes agrupamentos, e é possível perceber um padrão estabelecido para pelo menos três diferentes contextos pluviométricos: grupos 1; grupo 2 e 5 e grupo 3 e 4 . $\mathrm{O}$ agrupamento 1 , foi formado por seus pontos estarem fortemente associados aos 
elevados níveis de precipitação, evidenciando alterações pluviométricas controladas pelo fator climático. Por outro lado, as precipitações dos grupos 2 e 5 se posicionam em nós distantes dos demais nos dendogramas por conta de seus baixos níveis de precipitação, contudo, no grupo 2 a seca foi mais severa, sendo considerado um outlier no estudo. Os grupos 3 e 4 existem variações sazonais estabelecidas entre os grupos, onde foram observadas oscilações acima da média em maio, junho e julho para o grupo 3, já para o grupo 4 as oscilações ocorreram nos meses de janeiro, fevereiro e março.

Figura 7: Cluster correspondente ao município de Recife, no período de janeiro de 2000 a dezembro de 2018, Pernambuco, Brasil.

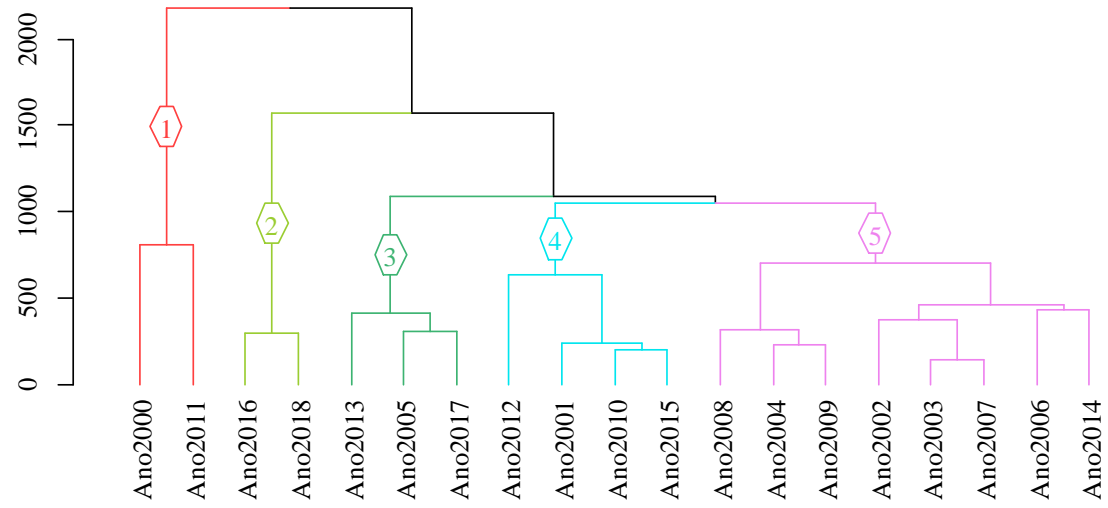

Fonte: Própria (2019).

Observando simultaneamente a Figura 7 e 8, é possível entender os tipos de dados que deram origem aos nós dos agrupamentos. Dessa forma, pode-se observar que os agrupamentos separaram os anos em altos, medianos e baixos níveis pluviométricos entre o período de 2000 a 2018.

Figura 8: Biplot do FPCA correspondente ao município de Recife, no período de janeiro de 2000 a dezembro de 2018, Pernambuco, Brasil. 
Fonte: Própria (2019).

Em relação a análise de variância funcional (Figura 9), verifica-se que houve diferença significativa entre Recife e Vitória do Santo Antão, esse fator está relacionado com a geolocalização dos municípios, pois Recife apresenta clima tropical quente úmido (Litoral) e Vitória de Santo Antão apresenta clima tropical quente subúmido e seco (Zona da Mata). No litoral, as chuvas estender-se ao longo de quase todo o ano com um pequeno período de seca,

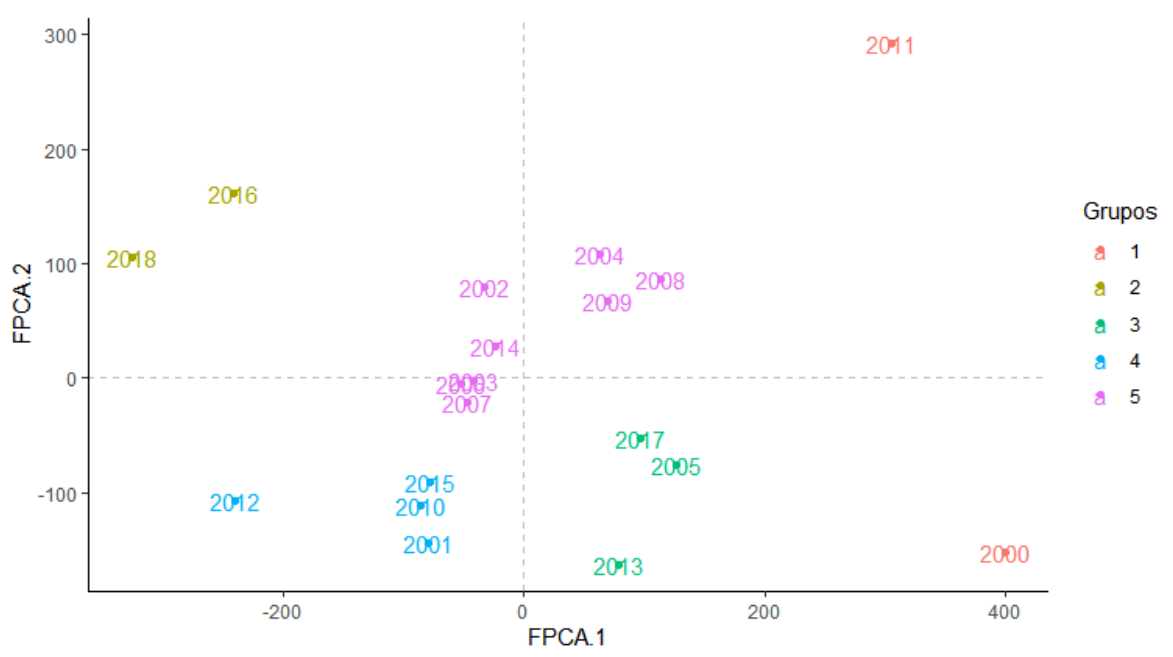

geralmente registrada na primavera. Além disso, o estado de Pernambuco sofre interferência direta da Zona de Convergência Intertropical Tropical (ZCIT), que proporciona um aumento de precipitação em todas áreas afetadas.

Figura 9: ANOVA Funcional dos municípios. 


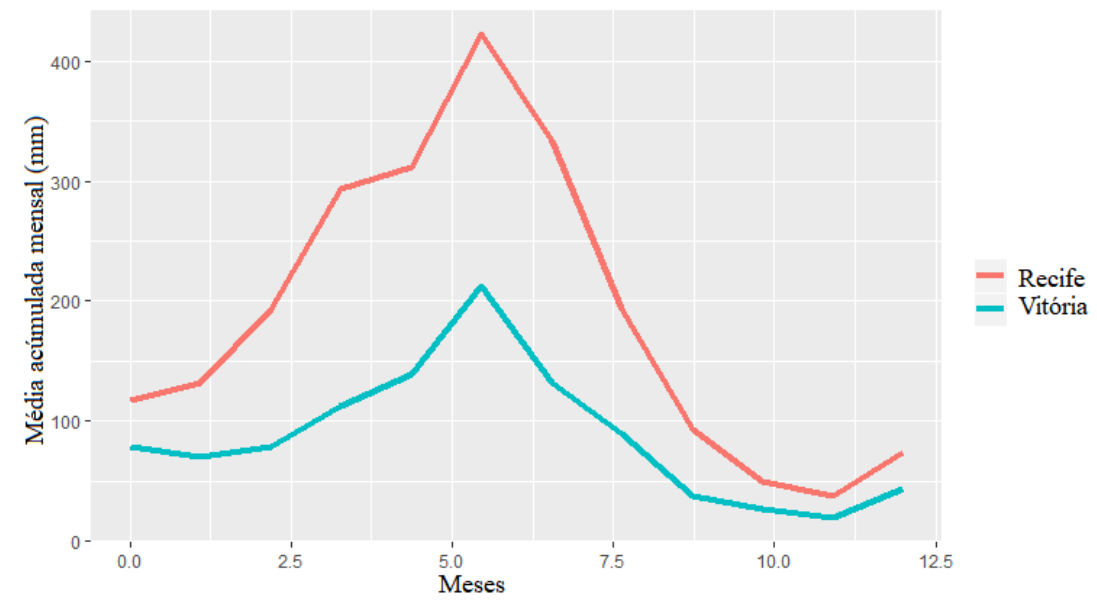

Fonte: Própria (2019).

\section{Conclusões}

Conclui-se que, análise de dados funcionais conseguiu captar o comportamento do fenômeno estudado indicando uma forte variação anual, exibindo-se diferenciações das precipitações entre os municípios, ou seja, possibilitou uma melhor compreensão da dinâmica da precipitação, considerando a natureza contínua do processo. Além disso, com base na literatura para fazer uma análise climatológica precisa-se necessariamente 30 anos de observações, contudo, a FDA analisa os padrões com quantidade inferiores de observações.

\section{Referências}

ALMAZROUI, M.; ISLAM, M. N.; JONES, P. D.; ATHAR, H.; RAHMAN, M. A. Recent climate change in the Arabian Peninsula: seasonal rainfall and temperature climatology of Saudi Arabia for 1979-2009. Atmospheric Research, v. 111, p. 29-45, 2012.

ALMEIDA, R.; REBELLO, E.; AMBRIZZI, T. Variabilidade de eventos extremos e identificação de tendências climáticas no litoral Norte do Brasil. In: XVI Congresso Brasileiro de Meteorologia. 2010.

ANDREOLI, R. V.; KAYANO, M. T. A importância relativa do Atlântico Tropical Sul e Pacífico Leste na variabilidade de precipitação do Nordeste do Brasil. Revista Brasileira de Meteorologia, v. 22, n. 1, p. 63-74, 2007.

ANDREOLI, R. V.; KAYANO, M. T.; GUEDES, R. L.; OYAMA, M. D.; ALVES, M. A. S. A influência da temperatura da superfície do mar dos Oceanos Pacífico e Atlântico na variabilidade de precipitação em Fortaleza. Revista Brasileira de Meteorologia, v. 19, n. 3, p. 337-344, 2004. 
User's Manual for Windows®. Springer Science \& Business Media, 2005.

CROUX C.; GAZEN A. R. High breakdown estimators for principal components: the projection-pursuit approach revisited. Journal of Multivariate Analysis, 95(1):206-226, 2005.

DAUXOIS, J.; POUSSE, A.; ROMAIN, Y. Asymptotic theory for the principal component analysis of a vector random function: some applications to statistical inference. Journal of multivariate analysis, v. 12, n. 1, p. 136-154, 1982.

DE LUCENA, L. R. R.; STOSIC, T.; FILHO, M. C. Avaliação da precipitação diária do estado de Sergipe utilizando análise de lacunaridade. Rev. Bras. Biom, v. 33, n. 2, p. 268$276,2015$.

DE SANTANA, L. I. T.; PEREIRA, M. M. A.; FREITAS, J. R.; CUNHA FILHO, M.; STOSIC, T. Aplicação da análise de recorrência na precipitação diária para o município de Vitória de Santo Antão/PE. Sigmae, v. 8, n. 2, p. 114-120, 2019.

DONA, G.; PREATONI, E.; COBELlI, C.; RODANO, R.; HARRISON, A. J. Application of functional principal component analysis in racewalking: an emerging methodology. Sports Biomechanics, v. 8, n. 4, p. 284-301, 2009.

DOS SANTOS, L. A. R.; LUCIO, P. S.; REBELLO, E. R. G.; BALBINO, H. T.; FORTES, L. T. G.; SALES, N. D. de; DINIZ, F. de A.; COSTA, M. C. G.; FERREIRA, D. B.; SALVADOR, M. A.; LEANDRO, I. V.; PAULA, T. P. de. Caracterização de extremos climáticos utilizando o software RClimdex. Estudo de caso: sudeste de Goiás. In: XIV Congresso Brasileiro de Meteorologia, Florianópolis, 2006.

GAO, H. O. Day of week effects on diurnal ozone/NOx cycles and transportation emissions in Southern California. Transportation Research Part D: Transport and Environment, v. 12, n. 4, p. 292-305, 2007.

GAO, H. O.; NIEMEIER, D. A. Using functional data analysis of diurnal ozone and NOx cycles to inform transportation emissions control. Transportation Research Part D: Transport and Environment, v. 13, n. 4, p. 221-238, 2008.

GÓRECKI, T.; SMAGA, L. fdANOVA: an R software package for analysis of variance for univariate and multivariate functional data. Computational Statistics, v. 34, n. 2, p. 571-597, 2019.

GUEDES, M. V.; SILVA, M. M. da. Situação das áreas de reserva legal e proteção dos recursos naturais em assentamentos rurais da Mata Meridional pernambucana. 2012. Dissertação de Mestrado. Universidade Federal de Pernambuco.

MOLION, L. C. B. Climatologia Dinâmica da região Amazônica: mecanismos de precipitação. Revista Brasileira de Meteorologia, v. 2, n. 1, p. 107-117, 1987.

MIRANDA, M. R. B. de. Análise da vulnerabilidade a inundações no médio curso do Rio 
Tapacurá Cidade de Vitória de Santo Antão-PE. 2015. Dissertação de Mestrado. Universidade Federal de Pernambuco.

NÓBREGA, R. S.; SANTIAGO, G. A. C. F. Tendência de temperatura na superfície do mar nos oceanos atlântico e pacífico e variabilidade de precipitação em Pernambuco. Mercator-Revista de Geografia da UFC, v. 13, n. 1, p. 107-118, 2014.

RAMSAY, J. O. Functional data analysis. Encyclopedia of Statistical Sciences, v. 4, 2004.

RAMSAY, J. O.; BOCK, R. D.; GASSER, T. Comparison of height acceleration curves in the Fels, Zurich, and Berkeley growth data. Annals of Human Biology, v. 22, n. 5, p. 413 426, 1995.

RAMSAY, J. O.; DALZELL, C. J. Some tools for functional data analysis. Journal of the Royal Statistical Society: Series B (Methodological), v. 53, n. 3, p. 539-561, 1991.

RAMSAY, J. O.; SILVERMAN, B. W. Functional Data Analysis. Springer-Verlag. New York, 1997.

RAMSAY, J.; HOOKER, G.; GRAVES, S. Functional Data Analysis with $\mathbf{R}$ and MATLAB. Use R, 1-207. [S.1.]: Springer, 2009. 208 p. ISSN 978-0-387-98184-0.

R CORE TEAM. R: A language and environment for statistical computing. R Foundation for Statistical Computing, Vienna, Austria. 2019. URL http://www.R-project.org/.

RENCHER, A. C. Methods of multivariate analysis. a john wiley \& sons, inc. publication. 2002.

ROPELEWSKI, C. F.; HALPERT, M. S. Global and regional scale precipitation patterns associated with the El Niño/Southern Oscillation. Monthly weather review, v. 115, n. 8, p. 1606-1626, 1987.

SAlGADO, C. M., da CRUZ SILVA, T., de SOUZA, G. C. A., \& de FREITAS, H. C. Caracterização temporal e espacial da precipitação no entorno do município de São Gonçalo (RJ) considerando a série histórica de 1968 a 2002. Sociedade \& Natureza, 19(1), 19-31, 2007.

SANTOS, P. V. dos; SANTOS, R. dos; COUTINHO, M. D. L. Detecção de mudanças climáticas através de índices pluviométricos diários no Estado de Pernambuco. Revista Brasileira de Geografia Física, v. 6, n. 4, p. 713-731, 2013.

SILVA, V. P., PEREIRA, E. R., de AZEVEDO, P. V., de SOUSA, F. D. A., \& de SOUSA, I. F. Análise da pluviometria e dias chuvosos na região Nordeste do Brasil. Revista Brasileira de Engenharia Agricola e Ambiental-Agriambi, v. 15, n. 2, 2011.

SILVA, R. O. B. da; MONTENEGRO, S. M. G. L.; SOUZA, W. M. de. Tendências de mudanças climáticas na precipitação pluviométrica nas bacias hidrográficas do estado de Pernambuco. Engenharia Sanitaria e Ambiental, v. 22, n. 3, p. 579-589, 2017. 
SILVA, R. M. de; SILVA, L. P. e; MONTENEGRO, S. M. G. L.; SANTOS, C. A. G. Análise da variabilidade espaço-temporal e identificação do padrão da precipitação na bacia do rio Tapacurá, Pernambuco. Sociedade\&Natureza, v. 22, n. 2, 2010.

SILVA, W. L.; DERECZYNSKI, C. Tendências observadas nos índices extremos diários de temperatura e precipitação na cidade do Rio de Janeiro. In: XVI Congresso Brasileiro de Meteorologia, Belém, Brasil. 2010.

SOUZA NETO, E. G. Análise de tendências e previsão das séries de precipitação do município de Vitória de Santo Antão - PE. 2017. Dissertação (Mestrado em Biometria e Estatística Aplicada) - Programa de Pós-Graduação em Biometria e Estatística Aplicada, Universidade federal Rural de Pernambuco, Recife - PE, 2017.

SOUZA, W. M. de; AZEVEDO, P. V. de. Índices de Detecção de Mudanças Climáticas Derivados da Precipitação Pluviométrica e das Temperaturas em Recife - PE. Revista Brasileira de Geografia Física, v. 5, n. 1, p. 143-159, 2012.

ULLAH, S.; FINCH, C. F. Applications of functional data analysis: A systematic review. BMC medical research methodology, v. 13, n. 1, p. 43, 2013.

XAVIER JR, S. F. A.; JALE, J. da S.; STOSIC, T.; SANTOS, C. A. C. dos; SINGH, V. P. An application of sample entropy to precipitation in Paraíba State, Brazil. Theoretical and Applied Climatology, v. 136, n. 1-2, p. 429-440, 2019.

WANG, J. L.; CHIOU, J. M.; MÜLLER, H. G. Functional data analysis. Annual Review of Statistics and Its Application, v. 3, p. 257-295, 2016.

WANG, S.; JANK, W.; SHMUELI, G.; SMITH, P. Modeling price dynamics in eBay auctions using differential equations. Journal of the American Statistical Association, v. 103, n. 483, p. 1100-1118, 2008. 